\title{
Role of Metabolic Shifts in Protection from Mutation Damage: Characterizing Mitochondrial Membrane Potential in C. Elegans Gas-1 Mutants
}

\author{
Lauren S. Muñoz-Tremblay
}

Portland State University

Follow this and additional works at: https://pdxscholar.library.pdx.edu/mcnair

Part of the Cancer Biology Commons, and the Genetics Commons Let us know how access to this document benefits you.

\section{Recommended Citation}

Muñoz-Tremblay, Lauren S. (2015) "Role of Metabolic Shifts in Protection from Mutation Damage: Characterizing Mitochondrial Membrane Potential in C. Elegans Gas-1 Mutants," PSU McNair Scholars Online Journal: Vol. 9: Iss. 1, Article 7.

https://doi.org/10.15760/mcnair.2015.101

This open access Article is distributed under the terms of the Creative Commons Attribution-NonCommercialShareAlike 4.0 International License (CC BY-NC-SA 4.0). All documents in PDXScholar should meet accessibility standards. If we can make this document more accessible to you, contact our team. 
Role of metabolic shifts in protection from mutation damage: Characterizing mitochondrial membrane potential in $\mathrm{C}$. elegans gas- 1 mutants

\author{
by \\ Lauren S. Muñoz-Tremblay
}

Faculty Mentor

Dr. Suzanne Estes

Citation: Muñoz-Tremblay, Lauren S. "Role of metabolic shifts in protection from mutation damage: Characterizing mitochondrial membrane potential in C.

elegans gas- 1 mutants". Portland State University McNair Scholars Online Journal, Vol. 9, 2015. 


\begin{abstract}
Many terminal human diseases are caused by mutations affecting mitochondrial functioning. Mitochondria are essential organelles responsible for producing cellular energy, adenosine triphosphate (ATP) via oxidative phosphorylation (OXPHOS) at mitochondrial electron transport chains (ETC). Proper ETC functioning relies on maintenance of the electrochemical gradient essential for energy production, known as mitochondrial membrane potential $(\Delta \boldsymbol{\Psi} M)$. The inner mitochondrial membrane is the site of the ETC and is most closely in contact with the enzymatic processes occurring within the mitochondrial matrix. Mutations affecting protein components of the ETC are especially troublesome for organelle health. ETC mutants commonly express altered $\Delta \psi \mathrm{M}$, as well as increased production of damaging reactive oxygen species (ROS), which are hypothesized to cause genomic damage and lasting mutation. The nematode $\mathrm{C}$. elegans is a practical model organism for investigating the phenotypic and genomic consequences of ETC mutations. Despite expressing higher levels of damaging ROS, the ETC protein complex 1 mutant, gas-1, expresses heritable mtDNA and nDNA mutation rates identical to those of wild type animals. I am using a mitochondria-targeted dye and fluorescence microscopy to quantify and compare $\Delta \psi M$ levels of the gas- 1 mutant and wild type animals. This work will provide a novel phenotypic characterization of this mutant and indicate whether decreased metabolic activity (e.g., reduced reliance on OXPHOS) is occurring in gas-1, and perhaps conferring protection from genomic degradation. Based on gas-1's characteristically low ATP production and high ROS production, I expect that $\Delta \psi \mathrm{M}$ will be higher in gas- 1 as compared to wild type levels.
\end{abstract}




\section{Introduction}

Mitochondria are arguably the most important organelle to cellular health and performance because they are the site of bioenergy metabolism for the entire cell. Glucose is digested and sent to the Citric Acid Cycle (TCA) located in the mitochondrial cytoplasm in the form of pyruvate, which is then metabolized to provide electrons in the form of $\mathrm{NADH}$ and $\mathrm{FADH}_{2}$ for use in oxidative phosphorylation (OXPHOS) occurring at the mitochondrial electron transport chain (ETC). The ETC is comprised of a series of proteins, organized into supercomplexes, located along the inner mitochondrial membrane. The movement of electrons through the complexes of the ETC produces more cellular energy in the form of ATP than glycolysis and the tricarboxylic acid cycle (TCA) cycle combined, and is powered by the constant movement of ions across the inner membrane, known as an electrochemical gradient, or the inner mitochondrial membrane potential $(\Delta \psi \mathrm{M})$.

Mutations in both nuclear DNA (nDNA) and mitochondrial DNA (mtDNA) can be responsible for coded dysfunction in a mitochondrion. Mutations affecting the ETC will consequently inhibit the production of ATP via OXPHOS and possibly other important regulatory functions of mitochondria. In addition to its central role in ETC and cellular function, maintenance of $\Delta \psi M$ is crucial for the process of mitochondrial fusion, and excessive fragmentation (fission) of mitochondria results when $\Delta \Psi \mathrm{M}$ is disrupted due to overwhelming proton translocator activity (Legros, 2002). Cycles of mitochondrial fission and fusion are crucial to overall cell health; however, only mitochondrial fusion is dependent upon $\Delta \Psi M$ (Legros, 2002). Because mitochondrial function is crucial for energy metabolism, dysfunctions within these organelles can lead to a broad range of diseases in animals, including Parkinson's disease in humans (Celardo, Martins, and Gandhi, 2014).

Complex 1 of the ETC is specifically responsible for the oxidation of NADH to lone electrons and protons and creating the initial proton gradient along the inner mitochondrial membrane, which initiates energy production through OXPHOS. This movement of ions is the main source of reactive oxygen species (ROS) due to electron leakage onto molecular oxygen, which is ever present due to the nature of this process (Hicks, Denver, and Estes, 2013). Although some ROS are critically important for molecular signaling, at high levels they are damaging to proteins, can cause morphological changes in mitochondria, disrupt $\Delta \psi M$, delay organismal development, and cause oxidative damage to DNA and lasting mutation (Shibuya and Tsujimoto, 2012). The idea that ROSinduced oxidative damage is an important source of somatic (non-gamete) or heritable (germline) mutation in nDNA or mtDNA genomes is controversial (Griffiths et al., 2000).

Altered metabolic processes were first observed in colorectal cancer cells (Warburg et al., 1956). The Warburg Effect is a phenomenon characterized by a shift in metabolism from primary use of OXPHOS to a less oxidatively stressful (i.e., lower ROS producing) but much less energetically efficient mixture of aerobic glycolysis and OXPHOS, which benefits rapidly proliferating cells (1956; Lunt and VanderHeiden, 2011). Ericson et al. (2012) measured the frequency of random de novo single base substitutions in the mtDNA of human colorectal cancer cells and discovered that tumor tissue had fewer C: G to T:A transition mutations (known to be associated with oxidative damage) compared to healthy colorectal tissue cells. Ericson et al. (2012) also showed that this decrease in mutation frequency was coupled with a metabolic shift from OXPHOS to anaerobic glycolysis, which was not witnessed in healthy colorectal cells. These findings suggest that the stability of the mitochondrial genome is increased in cancer compared to healthy cells, as a result of a decrease in ROS related mtDNA damage. Inhibited or suppressed energy production has also been observed in a wide variety of other diseases, including diabetes and neurodegenerative disorders (DeBerardinis, 2012). However, whether and to what extent altered or depressed metabolism can protect organisms from heritable (germline) mutations has not been studied.

C. elegans nematodes are becoming an increasingly important model system for studying mitochondrial dysfunction and its relationship with energy metabolism and mutation. These 
microscopic animals are easy to maintain, transparent, non-parasitic, and can be used for studying the effects of mutation on individuals and populations with relative ease. Among several ETC mutant alleles available for C. elegans, gas- 1 (fc21) is particularly well-studied. gas- 1 is a nuclear gene and gas- 1 ( $f(21)$ is a single base pair substitution that creates a hypomorphic allele in a protein component of ETC complex 1

(http://www.wormbase.org/species/c_elegans/gene/WBGene00001520?query=gas-1\#0-9e-3). gas- 1 expresses many altered physiological phenotypes such as increased dependence on ETC complex II to maintain metabolic homeostasis, decreased growth rate, decreased fecundity, a characteristic hypersensitivity to volatile anesthetics, increased levels of ROS, and decreased OXPHOS abilities as compared to that of wild type (Kayser et at., 2001). Despite the fact that gas1 mutants exhibit elevated levels of damaging ROS, previous studies performed by the D. R. Denver lab (Oregon State Univ.) have concluded that both mtDNA and nDNA mutation rates in gas1 are identical to those expressed by wild type animals (Estes pers. comm.). This result may indicate that ROS do not affect heritable mutation rates as previously thought. Based on these and previous findings, we believe that gas- 1 is experiencing a shift in metabolism synonymous with the Warburg Effect, and as a result, is gaining genomic protection from the mutagenic effects of high levels of ROS by reducing its dependence on energy production via the OXPHOS pathway (c.f., Ericson et al., 2012).

Previous phenotyping of gas- 1 has included various life-history and physiological traits (Kayser et al., 2001; Cairo et al., 1992; Estes et al., unpubl.), and studies of gene expression (Falk et al. 2008); however, $\Delta \Psi \mathrm{M}$ has not yet been characterized. Further investigation of gas- 1 is necessary to understand the effects of mutation on the ETC metabolic process, as well as the possibility of genomic preservation by means of altered metabolic pathways. By quantifying and comparing inner $\triangle \Psi \mathrm{M}$ of the mutant gas- 1 to that of wild type animals, it will be possible to gain an understanding of the effects of the gas- 1 mutation on energy production in C. elegans. Because $\Delta \psi M$ drives production of ATP via OXPHOS, these data allow us to detect any change in reliance of gas- 1 on this pathway and whether a shift in metabolism may be providing genomic protection for the gas- 1 mutant.

\section{Materials \& Methods}

Nematode culture conditions.

The C. elegans N2 strain and the nuclear mutant, gas-1(fc 21$)$, were obtained from the Caenorhabditis Genetics Center (University of Minnesota). Prior to experimentation, the D. R. Denver lab (Oregon State University) generated a completely isogenic gas- 1 mutant strain via 10 generations of backcrossing to the N2 strain during which the gas-1( $f c 21$ ) allele was tracked by PCR and Sanger DNA sequencing. Nematodes were thawed from a $-80^{\circ} \mathrm{C} \pm 2^{\circ} \mathrm{C}$ freeze and then kept on $60 \mathrm{~mm}$ Petri plates containing $15 \mathrm{~mL}$ of NGM-Lite agar that had been treated with $20 \mu \mathrm{g} / \mathrm{mL}$ streptomycin and seeded with OP50-1 Escherichia coli as a food source. Worms were regularly transferred to new plates with the use of a sterilized platinum scalpel to maintain small population sizes, avoid starvation, and decrease risk of bacterial contamination. Strains were stored in a dark, $20^{\circ} \mathrm{C}$ incubator. Populations no older than 30 days were used in data collection.

Fluorescence microscopy.

All methods were adapted from Hicks et al. (2012). When a sufficient number of worms from both strains had reached adulthood, their eggs were harvested to ensure age-synchronicity during future data collection. Age-synchronization was achieved by repeatedly rinsing samples with M9 buffer solution, dissolving adult worms with a 2:1 NaOH:bleach solution, and frequent centrifugation to promote sedimentation of egg pellets (Hicks et al., 2012). 
After the harvested eggs had matured on fresh plates for 24-58 hours, they were allowed to feed on fluorescently dyed E. coli for 24 hours (Hicks et al., 2012). A solution of $5 \mu \mathrm{L}$ of Chloromethyl-XRosamine (CMXROS) diluted with $495 \mu \mathrm{L}$ of M9 buffer solution was used per plate. CMXROS is a lipophillic, cationic, fluorescent dye that selectively binds to negatively charged components within the inner mitochondrial membranes of the worms (Pendergrass, Wolf, and Poot, 2004). The worms were then rinsed in M9 buffer solution and allowed to feed on un-dyed E. coli for 1 hour to pass any residual dyed E. coli from their intestinal tracts. Immediately prior to imaging, all worms were treated with a $20 \mathrm{mM}$ solution of paralytic levamisole. The pharyngeal bulb of each worm was imaged at a total magnification of $25 \mathrm{X}$, with the use of a fluorescence stereoscopic dissecting microscope (Leica MSV269). This neuromuscular organ was chosen to quantify $\Delta \psi M$ because of the large amount of localized mitochondria typically found within the boundaries of the bulb (Hicks, et al. 2012). It is also a site that contains minimal amounts of auto-fluorescent fat deposits, which decreases the likelihood of fluorescent interference during imaging.

Image processing and data analysis.

After images were collected, each bulb was manually encapsulated using ImageJ software (NIH). They were then analyzed by measuring mean and maximum fluorescence values and total area of each bulb in ImageJ. Statistical comparison of the two strains was achieved using a non-parametric Wilcoxon chi-square approximation (first data set) or a Student's t-test (second data set)

performed in JMP 9.0 (SAS). During image analysis, it became apparent that half of the nematodes comprising the first data set had not matured to the young adult stage prior to imaging. Because of this, a second set of data was collected and analyzed after the new sample had been verified to be young adult nematodes.

\section{Results}

Two sets of data from a total of three imaging sessions that took place in July and August were analyzed separately. Data were grouped in this fashion due to among-assay differences in nematode developmental stage (above). The first set of data (Figure 1 ) indicated that gas- 1 exhibited significantly lower levels of $\Delta \Psi \mathrm{M}$ than the wildtype $\mathrm{N} 2$ strain (Wilcoxon $\mathrm{X}^{2}=14.3, \mathrm{df}=1$, $\mathrm{N}=133, \mathrm{p}=0.0002$ ). Analysis of mean pixel intensity showed an identical pattern (data not shown). The second set of data (Figure 2.1) showed that gas- 1 trended toward higher $\Delta \psi M$ levels than the wildtype $\mathrm{N} 2$ strain ( $\mathrm{t}=-1.44, \mathrm{df}=1, \mathrm{~N}=77, \mathrm{p}=0.1547$ ), although the difference was not statistically significant. It was also observed that the wildtype strain had significantly larger pharyngeal bulbs than those of gas- $1(t=6.06, d f=1, N=77, p<0.0001)$ in the second data set (Figure 2.2). 


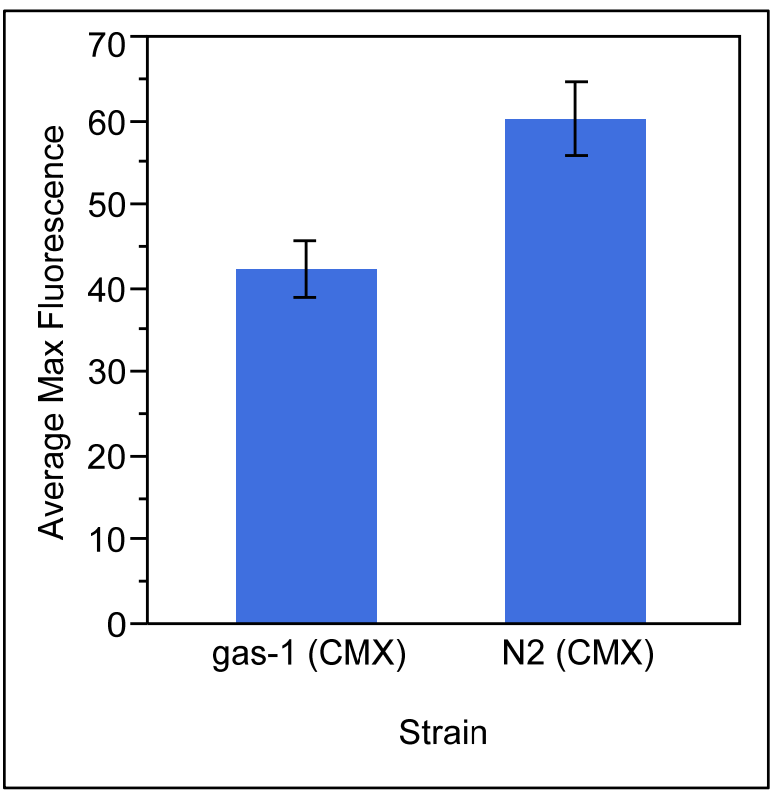

Figure 1: gas-1 strain exhibits lower levels of average maximum fluorescence within pharyngeal bulbs stained with MitoTracker CMXROS. Maximum

fluorescence is measured in pixel intensity, $Y$ axis. Error bars indicate one SEM $(S D / \sqrt{ } N)$.

\section{Conclusion:}

The first set of data $(N=133)$ indicated that gas- 1 exhibited lower $\Delta \psi M$ than that of wildtype N2 (Figure 1), although upon closer inspection it was determined that the animals were not imaged at the appropriate young adult stage necessary for accurate data collection. As C. elegans reaches maturity, it experiences a marked increase in the numbers of mitochondria accompanied by a shift in primary metabolic reliance from the TCA cycle/glyoxylate cycle to OXPHOS (Intermediary metabolism: Metabolic patterns during development and aging: 7-9, Wormbook, 2009); therefore, a lack of metabolic maturity could underlie the lower $\Delta \psi \mathrm{M}$ of gas- 1 compared to the wildtype N2 strain. Results from the second set of data ( $\mathrm{N}$ $=77$ ), which used a verified young adult population, suggested that the gas- 1 population expressed higher levels of $\Delta \psi M$ than that of the wildtype population (Figure 2.1), although the difference was not statistically significant. This observation could indicate that the gas- 1 mutation affecting ETC Complex 1 prohibits dissipation of $\Delta \psi M$ down the ETC, resulting in a highly polarized electrochemical gradient, which could also be contributing to gas-1's characteristically high ROS levels and abnormally low level of ATP production (Brand, 2000). The second set of data also revealed that gas- 1 had significantly smaller mean pharyngeal bulb areas than those of N2 (Figure 2.2). This large difference in bulb size between strains could be explained by the less efficient and less healthy metabolism of gas-1. Similarly, it is well-understood that the movement of the pharynx, or pharyngeal pumping, is recognized as a proxy for metabolic rate in $\mathrm{C}$. elegans (Collins, 2008).

Future work will conduct additional trials using a larger sample size will be used during data collection, and use confocal fluorescence microscopy, which will allow imaging of separate focal planes at higher magnification and increased resolution. This experiment will also be repeated using ten mutationaccumulation (MA) lines generated from either gas- 1 or N2 (in addition to the gas- 1 and N2 progenitor strains) to further explore the possibility of a shift in metabolism from OXPHOS to glycolysis among lines that have accumulate mutations under genetic drift against either the gas- 1 mutant or wildtype genetic background. Based on my preliminary findings, I would expect that gas$1 \mathrm{MA}$ lines would show further increased $\Delta \psi M$

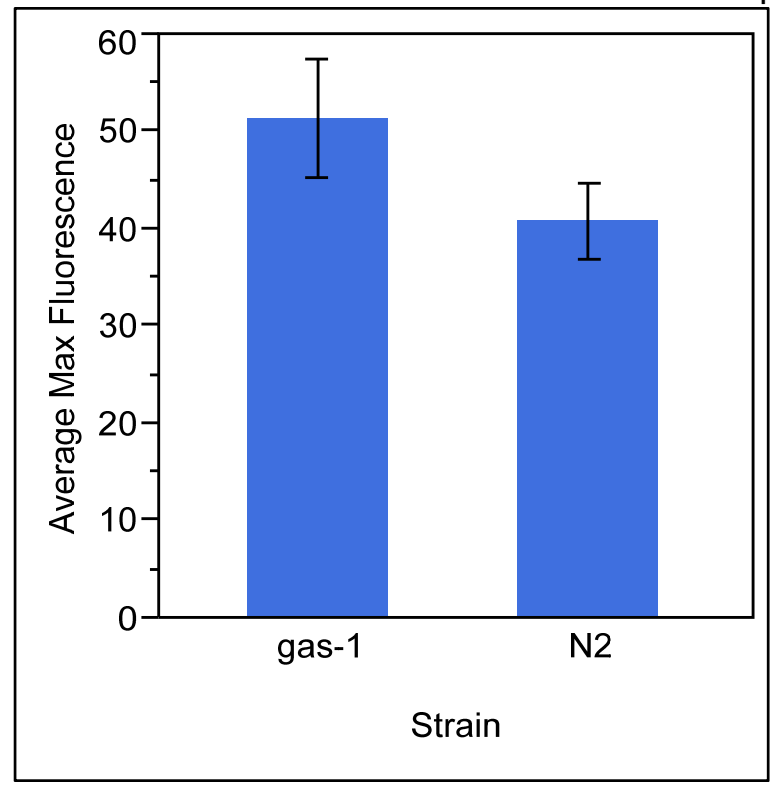


levels and would show greater variability in $\triangle \psi M$ levels from gas- 1 progenitor than N2 MA lines would show compared to N2 progenitor.

In contrast, I would expect N2 MA lines to

Figure 2.1: gas-1 strain exhibits higher average maximum fluorescence within pharyngeal bulbs stained with MitoTracker CMXROS than that of

wildtype. Maximum fluorescence is measured in

units of pixel intensity, $\mathrm{Y}$-axis. Error bars indicate one SEM $(\mathrm{SD} / \sqrt{ } \mathrm{N})$.

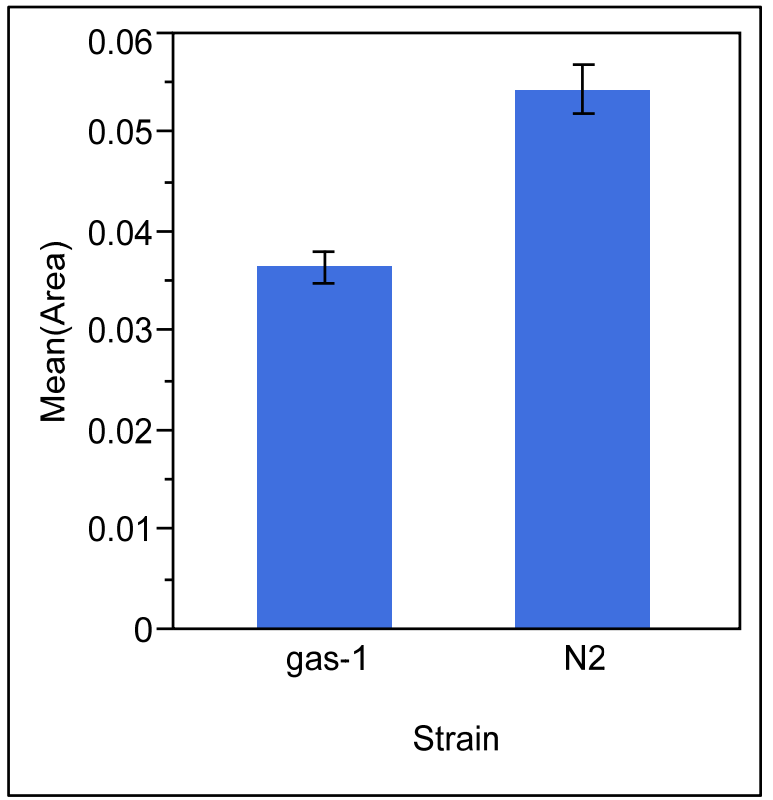

Figure 2.2: gas-1 strain exhibits smaller pharyngeal bulb area than that of wildtype N2 when CMXROS stained organs are manually encapsulated and measured with ImageJ data analysis software. Pharynx area is measured in pixels ${ }^{2}, \mathrm{Y}$-axis. Error bars indicate one SEM $(\mathrm{SD} / \sqrt{ } \mathrm{N})$. have similar or lower levels of $\Delta \psi M$ compared to N2 progenitor. 


\section{Bibliography}

1. Baer, Charles F. et al. "Comparative Evolutionary Genetics of Spontaneous Mutations Affecting Fitness in Rhabditid Nematodes." Proceedings of the National Academy of Sciences of the United States of America 102.16 (2005): 5785-5790. www.pnas.org. Web. 31 Mar. 2014.

2. "Barrick Lab : Procedure Bacterial Mutation Accumulation." N.p., n.d. Web. 31 Mar. 2014.

3. Bess, Amanda S. et al. "Mitochondrial Dynamics and Autophagy Aid in Removal of Persistent Mitochondrial DNA Damage in Caenorhabditis Elegans." Nucleic Acids Research 40.16 (2012): 7916-7931. http://www.dx.doi.org/10.1093/nar/gks532

4. Braeckman, Bart P. "Intermediary Metabolism." WormBook (2009): 1-24. CrossRef. Web. 10 Aug. 2014.

5. Brand, M. D. "Uncoupling to Survive? The Role of Mitochondrial Inefficiency in Ageing." Experimental Gerontology 35.6-7 (2000): 811-820. Print. http://dx.doi.org/10.1016/S0531-5565(00)00135-2

6. Celardo, I, L M Martins, and S Gandhi. "Unravelling Mitochondrial Pathways to Parkinson's Disease." British J ournal of Pharmacology 171.8 (2014): 1943-1957. Wiley Online Library. Web. 12 July 2014. http://dx.doi.org/10.1111/bph.12433

7. Chial, H. and Craig, J. "mtDNA and Mitochondrial Diseases." Nature Education 1 (2008):217.

8. DeBerardinis, Ralph J., and Craig B. Thompson. "Cellular Metabolism and Disease: What Do Metabolic Outliers Teach Us?" Cell 148.6 (2012): 1132-1144. www.cell.com. Web. 15 July 2014.

9. Ericson, Nolan G. et al. "Decreased Mitochondrial DNA Mutagenesis in Human Colorectal Cancer." PLoS Genet 8.6 (2012): e1002689. PLoS J ournals. Web. 12 July 2014. http://www.dx.doi.org/ 10.1371/journal.pgen.1002689

10. Estes, Suzanne, Patrick C. Phillips, et al. "Mutation Accumulation in Populations of Varying Size: The Distribution of Mutational Effects for Fitness Correlates in Caenorhabditis Elegans." Genetics 166.3 (2004): 1269-1279 http://dx.doi.org/10.1534/genetics.166.3.1269

11. ---. "Mutation Accumulation in Populations of Varying Size: The Distribution of Mutational Effects for Fitness Correlates in Caenorhabditis Elegans." Genetics 166.3 (2004): 1269-1279. http://dx.doi.org/10.1534/genetics.166.3.1269

12. Estes, Suzanne, Anna L. Coleman-Hulbert, et al. "Natural Variation in Life History and Aging Phenotypes Is Associated with Mitochondrial DNA Deletion Frequency in Caenorhabditis Briggsae." BMC Evolutionary Biology 11.1 (2011): 11. www. biomedcentral.com. Web. 15 July 2014.

13. Estes, Suzanne, Patrick C. Phillips, and Dee R. Denver. "Fitness Recovery and Compensatory Evolution in Natural Mutant Lines of C. Elegans." Evolution 65.8 (2011): 2335-2344. http://dx.doi.org/10.1111/j.1558-5646.2011.01276.x

14. MJ, Falk et al. "Metabolic Pathway Profiling of Mitochondrial Respiratory Chain Mutants in C. Elegans." Molecular genetics and metabolism 93.4 (2008): 388-397. http://dx.doi.org/10.1016/j.ymgme.2007.11.007

15. "fc21 (variation) - WormBase: Nematode Information Resource." N.p., n.d. Web. 12 July 2014.

16. Griffiths, Anthony JF et al. "Somatic versus Germinal Mutation." (2000): n. pag. http://www.ncbi.nlm.nih.gov/books/NBK21894/

17. Hicks, Kiley A. et al. "In Vivo Quantification Reveals Extensive Natural Variation in Mitochondrial Form and Function in Caenorhabditis Briggsae." PLoS ONE 7.8 (2012): e43837. PLoS J ournals. http://www.dx.doi.org/ 10.1371/journal.pone.0043837 
18. Hicks, Kiley A., Dee R. Denver, and Suzanne Estes. "Natural Variation in Caenorhabditis Briggsae Mitochondrial Form and Function Suggests a Novel Model of Organelle Dynamics." Mitochondrion 13.1 (2013): 44-51.

http://dx.doi.org/10.1016/j.mito.2012.12.006

19. Joyner-Matos, Joanna, Kiley A. Hicks, et al. "Evolution of a Higher Intracellular Oxidizing Environment in Caenorhabditis Elegans under Relaxed Selection." PLoS ONE 8.6 (2013): e65604. PLoS J ournals. Web. 31 Mar. 2014.

20. Joyner-Matos, Joanna, Laura C. Bean, et al. "No Evidence of Elevated Germline Mutation Accumulation Under Oxidative Stress in Caenorhabditis Elegans." Genetics 189.4 (2011): 1439-1447. http://dx.doi.org/10.1534/genetics.111.133660

21. Kayser, Ernst-Bernhard, Charles L. Hoppel, et al. "A Mutation in Mitochondrial Complex I Increases Ethanol Sensitivity in Caenorhabditis Elegans." Alcoholism: Clinical and Experimental Research 27.4 (2003): 584-592.

http://dx.doi.org/10.1111/j.1530-0277.2003.tb04394.x

22. Kayser, Ernst-Bernhard, Phil G. Morgan, et al. "Mitochondrial Expression and Function of GAS-1 in Caenorhabditis Elegans." J ournal of Biological Chemistry 276.23 (2001): 20551-20558. http://dx.doi.org/10.1074/jbc.M011066200

23. Keightley, Peter D. "Rates and Fitness Consequences of New Mutations in Humans." Genetics 190.2 (2012): 295-304. http://dx.doi.org/10.1534/genetics.111.134668

Kidane, Dawit, and Joann B. Sweasy. "Tipping the Balance in the Powerhouse of the Cell to 'Protect' Colorectal Cancer." PLoS Genet 8.6 (2012): e1002758. PLoS Journals. Web. 12 July 2014.

24. Legros, Frédéric et al. "Mitochondrial Fusion in Human Cells Is Efficient, Requires the Inner Membrane Potential, and Is Mediated by Mitofusins." Molecular Biology of the Cell 13.12 (2002): 4343-4354. http://dx.doi.org/10.1091/mbc.E02-06-0330

25. Lunt, Sophia Y., and Matthew G. Vander Heiden. "Aerobic Glycolysis: Meeting the Metabolic Requirements of Cell Proliferation." Annual Review of Cell and Developmental Biology 27.1 (2011): 441-464. http://dx.doi.org/10.1146/annurev-cellbio-092910-154237

26. "The Measurement and Analysis of Age-Related Changes in Caenorhabditis Elegans WormBook - NCBI Bookshelf." N.p., n.d. Web. 15 Aug. 2014.

27. Mullen, Andrew R. et al. "Reductive Carboxylation Supports Growth in Tumour Cells with Defective Mitochondria." Nature 481.7381 (2012): 385-388. http://www.dx.doi.org/ $10.1038 /$ nature 10642

28. Osada, Naoki, and Hiroshi Akashi. "Mitochondrial-Nuclear Interactions and Accelerated Compensatory Evolution: Evidence from the Primate Cytochrome c Oxidase Complex." Molecular Biology and Evolution 29.1 (2012): 337-346. http://dx.doi.org/10.1093/molbev/msr211

29. Paula, Wilson B. M. de et al. "Female and Male Gamete Mitochondria Are Distinct and Complementary in Transcription, Structure, and Genome Function." Genome Biology and Evolution 5.10 (2013): 1969-1977. http://dx.doi.org/10.1093/gbe/evt147

30. Pendergrass, W., N. Wolf, and M. Poot. "Efficacy of MitoTracker Green ${ }^{\mathrm{TM}}$ and CMXrosamine to Measure Changes in Mitochondrial Membrane Potentials in Living Cells and Tissues." Cytometry Part A 61A.2 (2004): 162-169.

http://dx.doi.org/10.1002/cyto.a.20033

31. Prezant, Toni R. et al. "Mitochondrial Ribosomal RNA Mutation Associated with Both Antibiotic-induced and Non-syndromic Deafness." Nature Genetics 4.3 (1993): 289294. http://dx.doi.org/10.1038/ng0793-289

32. Raamsdonk, Jeremy Michael Van et al. "Decreased Energy Metabolism Extends Life Span in Caenorhabditis Elegans Without Reducing Oxidative Damage." Genetics 185.2 (2010): 559-571. http://dx.doi.org/10.1534/genetics.110.115378 
33. Shibuya, Toshiharu, and Yoshihide Tsujimoto. "Deleterious Effects of Mitochondrial ROS Generated by KillerRed Photodynamic Action in Human Cell Lines and C. Elegans." J ournal of Photochemistry and Photobiology B: Biology 117 (2012): 1-12. http://dx.doi.org/10.1016/j.jphotobiol.2012.08.005

34. Taylor, Robert W., and Doug M. Turnbull. "Mitochondrial DNA Mutations in Human Disease." Nature Reviews Genetics 6.5 (2005): 389-402. http://dx.doi.org/10.1038/nrg1606

35. Warburg, Otto, and others. "On the Origin of Cancer Cells." Science 123.3191 (1956): 309314. Print. http://dx.doi.org/10.1126/science.123.3191.309

36. Wielgoss, Sébastien et al. "Mutation Rate Dynamics in a Bacterial Population Reflect Tension between Adaptation and Genetic Load." Proceedings of the National Academy of Sciences 110.1 (2013): 222-227.

http://dx.doi.org/10.1073/pnas.1219574110 